\title{
Un modèle rhéologique pour les argiles gonflantes
}

\section{J.C. ROBINET*}

M. PAKZAD*

\section{F. PLAS**}

* 1. Euro-Geomat Consulting, 51, route d'Olivet, Orléans 45100 France.

Tél. (33) 38. 66.22.20

Fax (33) 38.66.24.70

2. Centre de Recherche sur

la Matière Divisée, Groupe

Géomatériaux Université d'Orléans. CNRS, rue Léonard De Vinci. Orléans 45072, France

Tél. (33) 38.41.73.30

Fax (33) 38.41.70.63

** Agence nationale pour la gestion des déchets radioactifs (ANDRA), route du Panorama Robert Schuman, BP 38, Fontenay-aux-Roses 92266, France.

Tél. (33-16-1) 41.17.8178

Fax (33-16-1) 41. 17.84.08
Après avoir rappelé les mécanismes physico-chimiques de déformation des argiles saturées à l'échelle de la microstructure en condition isotherme, nous proposons un modèle élasto-plastique pour les argiles gonflantes. La formulation considère que pour les états de contrainte normalement consolidés, les déformations irréversibles proviennent essentiellement de la déformation des macroporosités, et sont traduites par la plasticité associative. En revanche pour les états de contrainte surconsolidés, des contraintes internes de répulsion non équilibrées prennent naissance au niveau des micro-porosités et provoquent la dilatance irréversible des macro-porosités. Le gonflement macroscopique induit par ce dernier mécanisme est formulé par un deuxième mécanisme plastique à écrouissage combiné, isotrope et cinématique. Le modèle rhéologique est formulé dans le cadre de la plasticité à écrouissage mécanique positif pour les états de contrainte normalement consolidés et à écrouissage "physico-chimique» pour les états de contrainte surconsolidés. Le modèle permet de représenter les caractéristiques fondamentales du comportement des argiles gonflantes saturées notamment l'existence d'une boucle d'hystérésis très marquée sur des chemins œedométriques cycliques et la présence d'une déformation volumique de contractance sur les chemins triaxiaux pour une argile gonflante surconsolidée. Le modèle comporte huit paramètres rhéologiques qui sont obtenus aisément à partir d'essais œedométriques et triaxaux. Enfin l'article présente des simulations sur différents chemins de sollicitation cycliques et les compare aux résultats expérimentaux réalisés sur l'argile de Boom. On constate que la prédiction du calcul est assez proche des résultats expérimentaux, ce qui confirme la possibilité de reproduire les caractéristiques les plus importantes du comportement des argiles gonflantes.

Mots clés : modélisation élastoplastique, argile gonflante, contrainte de répulsion, écrouissage physico-chimique.

\section{A constitutive model for expansive clays}

After remembering the physico-chemical mechanism of saturated clay in isothermal conditions, we propose an elastoplastic model for expansive clays. The formulation considers in normally consolidated states, an irreversible strain of microstructure due to macro-pores compaction. The irreversible macroscopic strains result from the deformation of microstructures are described with associative plastic law. In the overconsolidated states, the repulsive stresses provoke an irreversible dilatancy of macro-pores. The macroscopic swelling of the last phenomenon is formulated by the second plastic mechanism with the combined isotropic and kinematics hardening. The rheological model schematises the normally consolidated states by mechanical hardening and the overconsolidated states by $\alpha$ physico-chemical $x$ hardening. The model is able to describe the fundamental characteristic of saturated expansive clays, especially the hysteresis loop in odometer tests and the existence of the volume change in triaxial tests at over-consolidated states. The model needs eight parameters that must be identified by means of the odometer and triaxial tests. Finally, this paper shows the predictions of model for the different tests with the experiments performed on Boom clay. The comparison between experimental results and model confirm the behaviour of the expensive clays.

Key words : elasto-plastic modelisation, expansive clay, repulsion stress, physico-chemical hardening. 


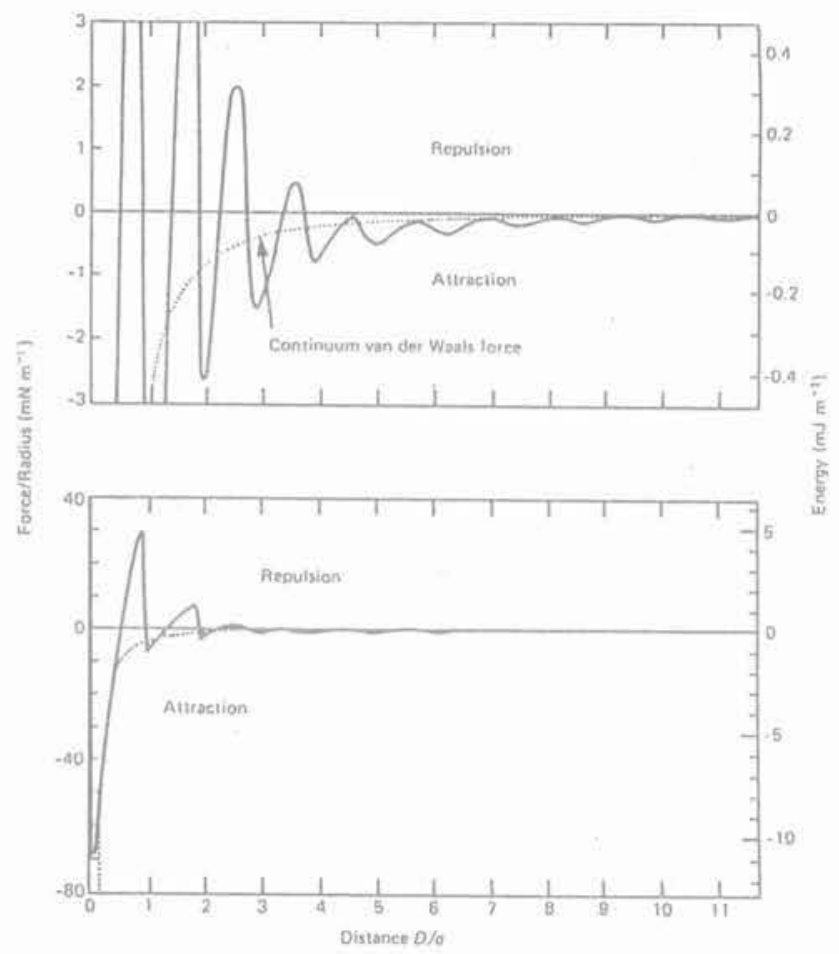

(a)

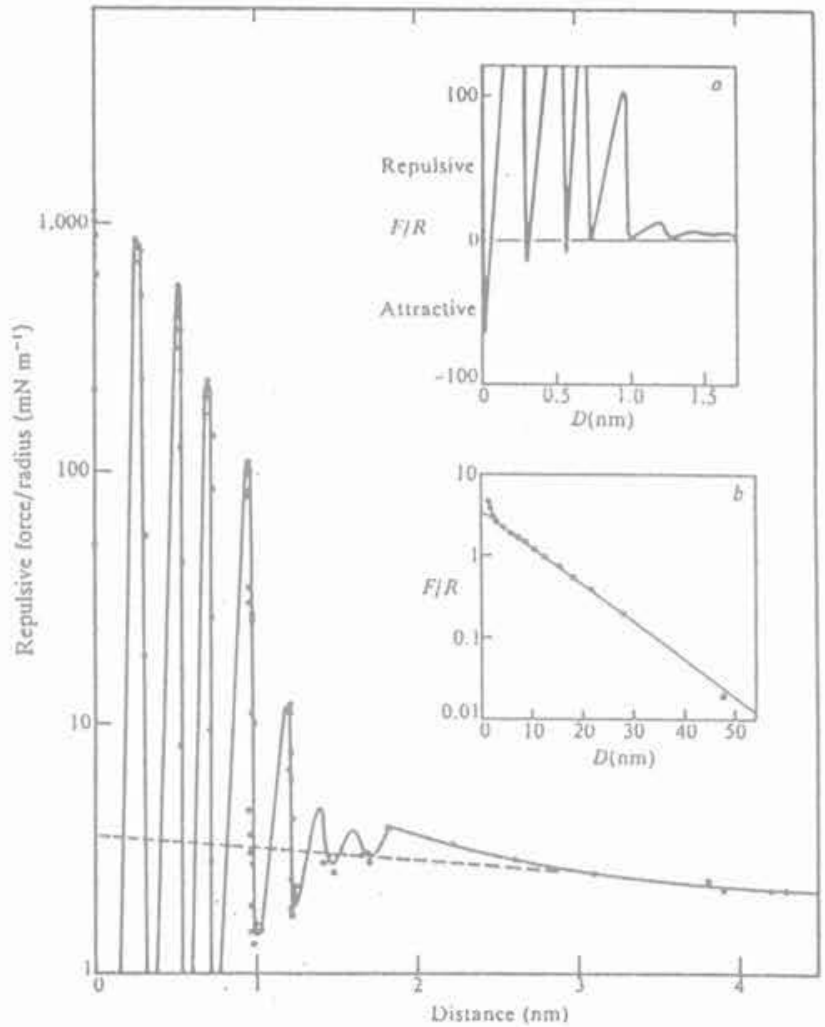

(b)

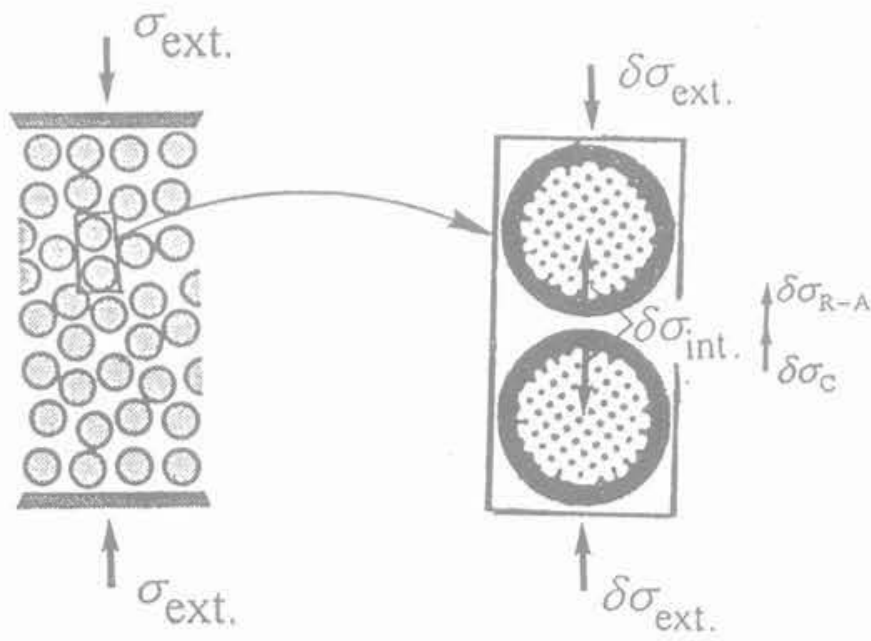

$$
\delta \sigma_{\text {ext. }}=\delta \sigma_{\text {int. }}=\delta \sigma_{\mathrm{C}}+\delta \sigma_{\mathrm{R}-\mathrm{A}}
$$

(c)

Distribution des forces internes (répulsion et attraction) entre deux disques de mica de rayon $\mathrm{R}=1 \mathrm{~cm}$ en fonction de leur espacement et des types de fluide: (a) non-aqueux (Horn et Israelachvili, 1981); (b) aqueux (Israelachvili et Pashley, 1983); (c) aqueux avec illustration des contraintes internes.

The repulsive and attraction forces distribution between two curved mica surfaces of radius $\mathrm{R}=1 \mathrm{~cm}$ as a fonction of distance and the type of liquids: (a) nonaqueous (Horn and Israelachvili 1981); (b) aqueous (Israelachvili and Pashley 1983); (c) aqueous with illustration of the intern stress. 


\section{Introduction}

Les argiles saturées sont constituées de trois phases: i) solide dont l'unité de base est le feuillet, ii) l'eau adsorbée interne et externe, et iii) l'eau libre.

L'organisation des espaces poraux associée à la nature minéralogique des arqiles joue un rôle fondamental dans le comportement mécanique. L'ensemble des structures solides délimite des espaces poraux de tailles différentes associés à des pourcentages volumiques spécifiques des différents types d'eau. La phase solide s'organise en structure croissante: le feuillet, l'empliement de feuillets, la particule, et l'agrégat. Ainsi l'espace inter-foliaire prend naissance à l'intérieur de l'empliement des feuillets d'argile, sur une distance moyenne de 15 à $25 \AA$. La distance inter-foliaire et le nombre de feuillets empilés sont variables avec la nature minéralogique des matériaux et de la charge sur les feuillets à travers les cations inter-foliaires. L'ensemble des feuillets définit sur une structure en forme de fibre. Les dimensions moyennes des pores inter-particuliaires sont d'environ 200 à $1500 \AA$ pour une argile à faible densité (Touret et Pons, 1989) et 10 à $25 \AA$ pour une argile à forte densité (Pusch, 1982). L'agrégat est constitué par l'assemblage de différentes fibres argileuses sous forme de grains, la distance moyenne des pores inter-agrégats est de l'ordre de 1.5 à $16 \mu \mathrm{m}$. Ces trois espaces poraux sont associés à un type d'eau dominant (Pons et al., 1982): i) l'eau libre dans les espaces inter-agrégats; ii) l'eau adsorbée interparticulaire; iii) l'eau adsorbée inter-foliaire.

L'eau adsorbée qui acquière une structure orientée, est peu sensible au gradient hydraulique; elle se comporte plutôt comme un solide et sa densité peut atteindre 1,4 (Martin, 1960; Low, 1979). Des essais de cisaillement sur les micro-structures (feuillets argileuxeau adsorbée) ont mis en évidence un comportement indépendant de la vitesse de sollicitation (Israealachvili et Adams, 1978). Par ailleurs, on observe que la contrainte de cisaillement maximale croit avec la diminution du nombre de couches d'eau adsorbée, ce qui amène à considérer que le comportement de l'eau adsorbée est similaire à celui d'un milieu granulaire plastique. L'argile saturée peut alors être réduite à un milieu biphasique: phase solide argileuse-eau adsorbée, et eau libre.

Dans le cas précis des argiles gonflantes (smectite); la structure de base est formée d'un empilement ionocovalent de trois couches élémentaires oxygénées et hydroxylées: une couche à coordinence octaédrique autour d'ions $\mathrm{Al}^{3+}$ ou $\mathrm{Mg}^{2+}$, comprise entre deux couches à coordinence tétraédrique autour d'ions $\mathrm{Si}^{4}$. Les substitutions ioniques confèrent au feuillet argileux, une charge négative ( $\mathrm{Al}^{3+}$ substitué par $\mathrm{Mg}^{2+}$ pour la montmorillonite). Ainsi les smectites ont une charge de 0,4 à 1,2 électrons par maille, alors que pour la vermiculite, les charges sont de 1,2 à 1,8 électrons par maille. C'est précisément cette charge négative qui est à l'origine de l'écartement des feuillets argileux dans le solvant polaire et qui leur confère le caractère gonflant.

\section{Inventaire des mécanismes microscopiques responsables des déformations macroscopiques irréversibles}

Le comportement d'une structure élémentaire feuillet argileux-eau correspondant aux milieux argileux gonflants ou non-gonflants a été étudié par Israelachvili et al. (1981 et 1983) à travers la distribution des forces d'interaction entre deux plaques de mica immergées dans un liquide: i) non aqueux, ii) aqueux (Fig. 1).

Dans le premier cas, les argiles non-gonflantes correspondent à un équilibre des forces internes de répulsion et d'attraction; elles sont étudiées en prêsence d'un liquide inerte caractérisé par les molécules non polaires (Fig. 1a). Pour une telle configuration, on constate que les contraintes résultantes d'attraction et répulsion sont négligeables. Les contraintes extérieures sont alors équilibrées par les contraintes de contact entre les molécules d'eau. En revanche, dans le second cas, les argiles gonflantes sont caractérisées par un excès des forces de répulsion par rapport aux forces d'attraction (Fig. 1b). Ces forces de répulsion dépendent très fortement de la concentration molaire du fluide interstitiel jusqu'à une distance minimale de $10 \AA$. Pour des distances entre plaques inférieures à $10 \AA$, les forces répulsives oscillent en augmentant très fortement. Il en résulte que pour une argile gonflante, les contraintes extérieures de chargement sont équilibrées par la superposition des contraintes de contact et des contraintes de répulsion (Fig. 1c).

L'assemblage des structures élémentaires (feuilleteau) forme un milieu désordonné où cohabitent trois types d'espaces poraux : inter-foliaire, inter-particulaire et inter-agrégats. L'évolution de la distribution des pores (en taille et en pourcentage) au cours de la consolidation d'une boue d'argile gonflante de Boom (Tableau I) fait apparaittre que la micro-porosité reste inchangée, en revanche on observe une diminution du pourcentage des macro-porosités (espaces inter-agrégats) (Fig. 2). Il en résulte que la pression de répulsion générée dans les espaces inter-foliaires et inter-particulaires reste constante pour les valeurs des

TABLEAUH Les caractéristiques physiques et minéralogiques de l'argile de Boom (Baldi et al. 1987).

Physical properties and mineralogic composition of Boom clay (Baidi et al. 1987).

\begin{tabular}{l|l}
\hline - Teneur en eau initiale & $23,9 \%$ \\
- L'indice des vides initial & 0,70 \\
\hline - Densité de grain & 2,70 \\
- Densité humide & 1,99 \\
- Densité sèche & 1,55 \\
- Surface spécifique & $53-177 \mathrm{~m}^{2} / \mathrm{g}$ \\
- Capacité d'échange de base & $20-40 \mathrm{meq} / 100 \mathrm{~g}$ \\
- Limite dé liquidité & $59-76 \%$ \\
- Limite de plasticité & $22-26 \%$ \\
- Indice de plasticité & $27-50 \%$ \\
& \\
Composition: & \\
- Smectite & $22 \%$ \\
- Illite & $20 \%$ \\
- Kaolinite & $30 \%$ \\
- Quartz & $28 \%$ \\
\hline
\end{tabular}



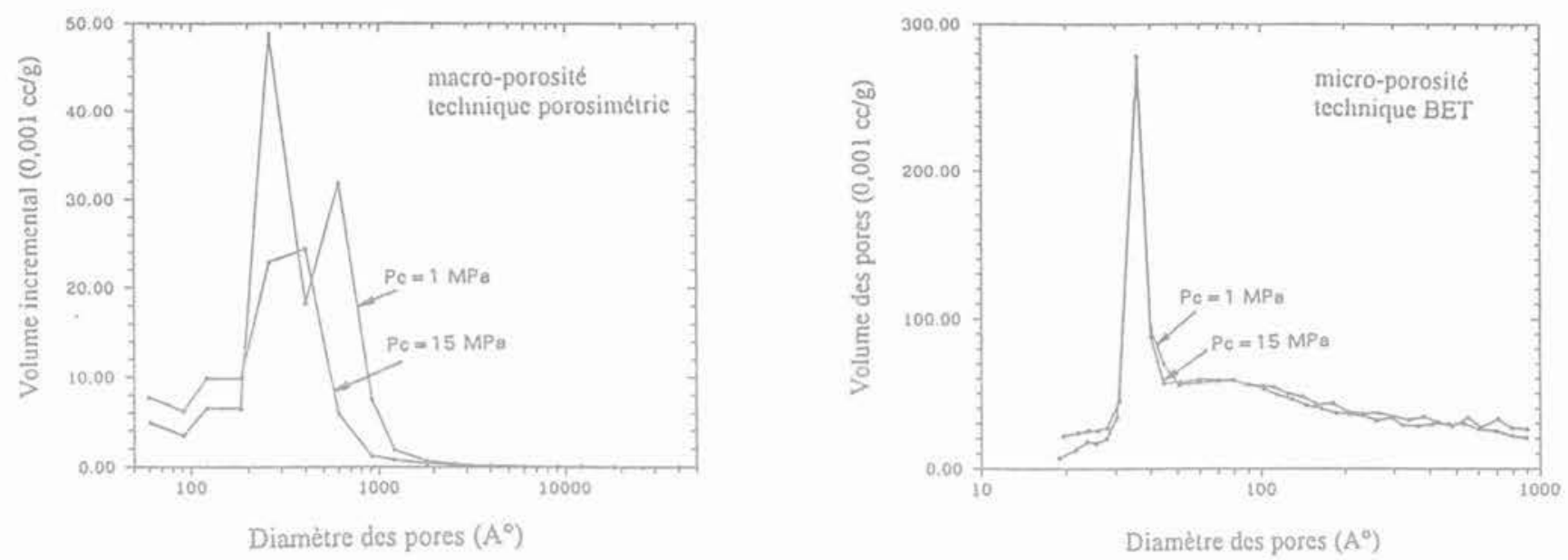

FIG.2 Essai de porosimétrie et distribution de la porosité en fonction de la pression de consolidation sur I'argile de Boom (Belanteur et Al-Mukhtar 1993).

Porosimetry test and pore size distribution as a fonction of consolidation pressure in Boom Clay (Belanteur and AlMukhtar 1993).

contraintes supérieures à $1 \mathrm{MPa}$. Les variations des contraintes extérieures produisent alors une variation des contraintes de contact $\left(\delta \sigma_{\text {extérieure }}=\delta \sigma_{\text {contact }}\right)$. Ce phénomène est illustré sur des essais œdometriques réalisés sur une argile gonflante de type smectite (Tableau II) et sur une kaolinite (Tableau III) qui ne possède pas un excédent des forces de répulsion. En effet les pentes de consolidation sont presque identiques pour les deux matériaux (Fig. 3).

Par ailleurs, on constate sur la figure 3 que la différence la plus significative du comportement est observée en décharge. La smectite possède un gonflement

\begin{tabular}{ll} 
TABLsau II & $\begin{array}{l}\text { Les caractéristiques physiques et } \\
\text { minéralogiques de la Smectite. } \\
\text { Physical properties and mineralogic } \\
\text { composition of smectite. }\end{array}$ \\
\hline - Densité de grain & 2,75 \\
- Surface spécifique & $426 \mathrm{~m}^{2} / \mathrm{g}$ \\
- Capacité d'échange de base & $64 \mathrm{meq} / 100 \mathrm{~g}$ \\
- Limite de liquidité & $112 \%$ \\
- Limite de plasticité & $50 \%$ \\
- Indice de plasticité & $62 \%$ \\
- Teneur en eau finale & \\
(décharge à 25 MPa) & $46 \%$ \\
Composition: & \\
- Smectite/Kaolinite (50/50) & $80 \% \pm 2$ \\
- Kaolinite & $4 \% \pm 2$ \\
- Geothite & $6 \% \pm 1$ \\
- Calcite & $2 \% \pm 1$ \\
- Quartz & $6 \% \pm 1$ \\
\hline
\end{tabular}

TABLEAU III Les caractéristiques physiques et minéralogiques de la Kaolinite. Physical properties and mineralogic composition of kaolinite.

\begin{tabular}{ll}
\hline $\begin{array}{l}\text { - Surface spécificue } \\
\text {-Teneur en eau finale } \\
\text { (décharge à 13 MPa) }\end{array}$ & $10,3 \mathrm{~m}^{2} / \mathrm{g}$ \\
& $31 \%$ \\
Composition: & \\
- Kaolinite & $86 \% \pm 3$ \\
- Quartz & $6 \% \pm 1$ \\
- Feldspar et Mica & $8 \% \pm 1$
\end{tabular}

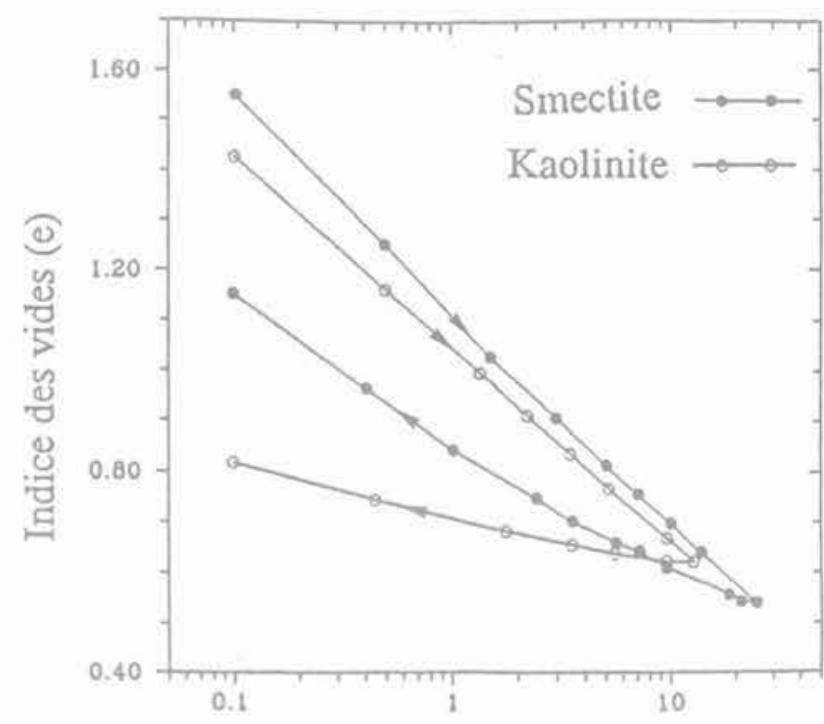

Contrainte axiale (MPa)

9G. 3 Essai œdométriques sur la Smectite et la Kaolinite (Pakzad 1993).

Edometer tests in smectite and kaolinite (Pakzad 1993).

beaucoup plus important que la kaolinite. Cette différence de comportement est dûe à l'existence des charges négatives dans les argiles gonflantes qui sont à l'origine du gonflement inter-folaire (Van Damme et al., 1987). La diminution de la contrainte mécanique externe entraîne une augmentation de la distance moyenne entre les feuillets. Il en résulte une diminution de la pression de répulsion, jusqu'à ce qu'un nouvel état d'équilibre soit atteint. En décharge, l'excès des contraintes de répulsion est équilibré par la déformation des micro-porosités qui induit alors une augmentation du pourcentage des macro-porosités. Ces déformations jouent en quelque sorte le rôle de catalyseur pour les déformations macroscopiques irréversibles liées au gonflement des macro-porosités. Dans une 
telle configuration, la variation des contraintes extérieures est compensée par la variation des contraintes de répulsion et d'attraction $\left(\delta \sigma_{\text {extérieure }}=\delta \sigma_{\mathrm{R} \sim \mathrm{A}}\right)$. Pour décrire le comportement contrainte-déformation des argiles gonflantes, Mitchell (1976) et Sdriharan (1990) ont étendu le concept des contraintes effectives de Terzaghi et supposent que les déformations du milieu sont contrôlées par les trois états de contraintes: $\sigma^{\prime}$, contrainte effective de contact; $\mathrm{U}_{\mathrm{g}}$ pression interstitielle; et $\sigma_{\mathrm{B}-\mathrm{A}}$, contrainte interne résultante des répulsions et des attractions : $\sigma=\sigma_{\mathrm{c}}^{\prime}+\mathrm{U}_{\mathrm{f}}+\sigma_{\mathrm{R}-\mathrm{A}}$ :

L'aspect mécanique du comportement des argiles gonflantes a été principalement étudié à travers la pression du gonflement définie comme la charge extérieure ne générant aucune déformation du matériau au cours de son hydratation. Toutefois, il a été constaté que la pression de gonflement mesurée au laboratoire dépendait fortement du chemin de sollicitation (Sridharan et al., 1982). Il en résulte que le comportement contraintedéformation en décharge d'une argile gonflante ne peut pas être modélisé par l'élasticité. En revanche les concepts de la plasticité peuvent être étendus aux chemins de décharge.

\section{3}

\section{Caractéristiques mécaniques des argiles gonflantes}

Dans cette étude, les caractéristiques mécaniques des argiles gonflantes sont déterminées à partir des chemins œdométriques et triaxiaux réalisés sur l'argile naturelle de Boom et sur une smectite remaniée. A partir des essais œdométriques, on observe les phénomènes suivants: la pente de consolidation dans l'essai œdométrique $\left(\lambda_{1}\right)$, sur l'argile gonflante type smectite, est identique à celle d'une argile non-gonflante type kaolinite. En revanche, la pente de gonflement $\left(\lambda_{2}\right)$ en décharge œdométrique, est plus grande pour la smec- tite que pour la kaolinite. De plus pour l'argile gonflante cette pente augmente avec la pression de consolidation, $\lambda_{2}=\mathrm{f}\left(\mathrm{P}_{\mathrm{C}}\right)$, (Fig. 4). Par ailleurs, le comportement œdométrique cyclique des argiles gonflantes est caractérisé par l'existence de grandes boucles d'hystérésis et par un effacement de la mémoire du matériau lorsque les états de contrainte deviennent normalement consolidés. Enfin les différences les plus significatives du comportement d'une argile gonflante par rapport à une argile non-gonflante sur des chemins triaxiaux sont observées pour les états de contrainte surconsolidés, par des déformations volumiques de contractance, alors qu'une kaolinite présente une dilatance très marquée.

\section{4}

\section{Formulation du modèle}

Les déformations des textures des argiles gonflantes proviennent: i) des variations des contraintes de contact pour les états normalement consolidés, ii) des variations des contraintes de répulsion et d'attraction pour les états surconsolidés. Ces variations des contraintes sont associées à deux mécanismes plastiques traduits par deux surfaces de charge: $F_{c}$ correspondant à la variation des contraintes de contact, $\mathrm{F}_{\mathrm{R}-\mathrm{A}}$ résultant des variations des contraintes de répulsion et d'attraction.

\section{1}

\section{La surface de charge correspondante aux contraintes de contact $\left(F_{c}\right)$}

A l'état normalement consolidé le modèle est défini sur la base de Cam-Clay modifié et repose sur les hypothèses suivantes:

H1: La partie réversible du comportement est décrite par l'élasticité non-linéaire:

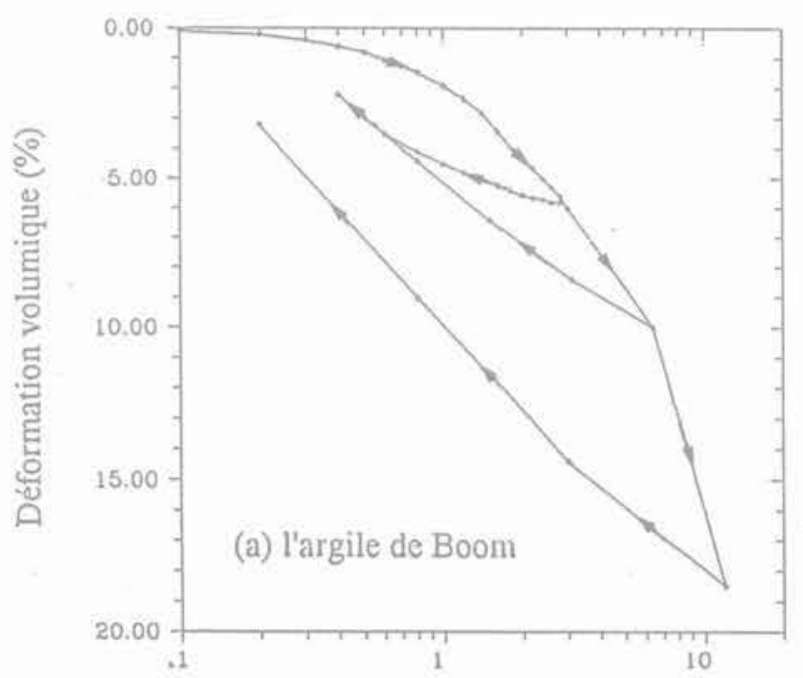

Contrainte axiale (MPa)

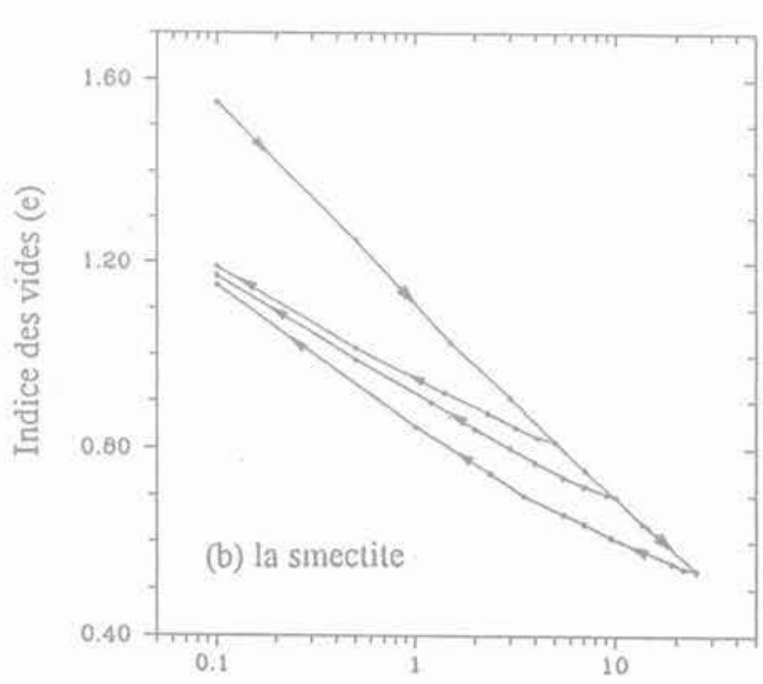

Contrainte axiale (MPa)

FIG.4 Essais isotropes et œdométriques à différente contrainte de décharge sur: (a) l'argile de Boom (Baldi et al. 1987); (b) la smectite (Pakzad 1993).

Isotropic and cedometer tests at different unloading stress in: (a) Boom clay (Baldi et al. 1987); (b) Smectite (Pakzad 1993). 


$$
\dot{\varepsilon}_{i j}^{e}=\frac{\dot{q}}{3 G}+\frac{\dot{P}^{\prime}}{3 K} \delta_{i j} \text { avec } K=K_{a} P_{a}\left(\frac{P^{\prime}}{P_{a}}\right)^{n} ; G=G_{a} P_{a}\left(\frac{P^{\prime}}{P_{a}}\right)^{n}
$$

$\mathrm{P}^{\prime}$ et $\mathrm{q}$; contraintes moyennes et déviatoires

$\mathrm{K}_{\text {}}$ et $\mathrm{G}_{\mathrm{a}}$ : les modules élastiques initiaux (volumiques et deviatoires)

$\mathrm{P}_{\mathrm{s}} \quad$ : pression atmosphérique

n : paramètre du modèle

H2: L'état du matériau est parfaitement défini par son état de contraintes $\left(\mathrm{P}^{\prime}, \mathrm{q}\right)$ ) et la seule variable d'écrouissage est $P_{c e}$

avec:

$$
\mathrm{P}_{\mathrm{Cf}}=\mathrm{P}_{\mathrm{CO}} \exp \beta_{1} \varepsilon_{\mathrm{v}}^{\mathrm{p}}
$$

$\mathrm{P}_{\mathrm{CO}} \quad$ : presion de préconsolidation

$\varepsilon_{v}^{p} \quad$ : la déformmation plastique volumique

$3_{1}:$ module de compressibilité plastique $=\frac{1+e_{0}}{\lambda_{1}-K}$

$\lambda_{\text {r }}$ et $\kappa$ : les pentes élasto-plastique et élastique du matériau

$\mathrm{e}_{0} \quad$ : l'indice des vides initial.

H3: La rupture est caractérisée par l'état critique: $\dot{\varepsilon}_{\mathrm{v}}^{\mathrm{p}}=0$ et $\mathrm{q}=\mathrm{MP}$ ?

$M$ est relié à l'angle de frottement du matériau, $\phi$.

$$
\begin{aligned}
& M=M_{C}=\frac{6 \sin \phi}{3-\sin \phi} \text { en compression } \\
& M=M_{E}=\frac{-6 \sin \phi}{3+\sin \phi} \text { en extension. }
\end{aligned}
$$

H4: La surface de charge est définie par l'expression:

$$
F_{C}=q^{2}+M^{2} P^{\prime}\left(P^{\prime}-P_{C f}\right) R^{2}(\theta)=0
$$

$R(\theta)$ est une fonction de forme qui permet de définir un critère de rupture proche de celui de mohr-Coulomb.

$$
R(\theta)=\frac{\sqrt{3}}{2} \frac{k}{k \sin \theta+\cos \left(\frac{\pi}{6}-\theta\right)}
$$

k est un paramètre qui définit le degré de convexité de la surface de rupture.

H5: Les taux de déformation totale sont la contribution d'une partie réversible et d'une partie irréversible:

$$
\hat{\varepsilon}_{i j}=\dot{\varepsilon}_{i j}^{e}+\dot{\varepsilon}_{i j}^{p}
$$

Les taux de déformations plastiques sont définis par:

$$
\dot{\varepsilon}_{i j}^{p}=d \lambda \cdot \frac{\partial F_{C}}{\partial \underline{\sigma}_{i j}^{\prime}}
$$

d $\lambda$ est le multiplicateur de plasticité, déterminé par la condition de consistance :

$$
\mathrm{d} \lambda=\frac{\frac{\partial \mathrm{F}_{\mathrm{C}}}{\partial \underline{\sigma}^{\prime}} \underline{\underline{\mathrm{C}}} \dot{\underline{\varepsilon}}}{\frac{\partial \mathrm{F}_{\mathrm{C}}}{\partial \underline{\sigma}^{\prime}} \underline{\underline{\mathrm{C}}} \frac{\partial \mathrm{F}_{\mathrm{C}}}{\partial \underline{\sigma}^{\prime}}-\frac{\partial \mathrm{F}_{\mathrm{C}}}{\partial \mathrm{F}_{\mathrm{Cf}}} \frac{\partial \mathrm{P}_{\mathrm{Cf}}}{\partial \varepsilon_{\mathrm{v}}^{\rho}} \frac{\partial \mathrm{F}_{\mathrm{C}}}{\partial \mathrm{P}^{\prime}}}
$$

$\underline{C}$ et $\sigma^{\prime}$ sont la matrice d'élasticité et le vecteur des contraintes effectives.

\section{2}

\section{Surface de charge associée aux contraintes de répulsion $\left(F_{R-A}\right)$}

Pour les états de contrainte surconsolidés, le couplage entre les déformations des micro-porosités et des macro-porosités, induit par les déséquilibres entre les contraintes internes et externes, est à la base du deuxième mécanisme plastique. Ce mécanisme est associé à une surface de charge appelée $F_{R-A}$ à plasticité non-associative et à écrouissage combiné isotrope et cinématique. En décharge, cette surface $F_{\mathrm{H}-\mathrm{A}}$ reste au contact avec la surface $F_{\text {c }}$ et son point de contact reste fixe. En recharge, ce mécanisme plastique est réactivé jusqu'à l'obtention des états de containte normalement consolidés, caractérisés par le contact entre les deux surfaces de charge $F_{C}$ et $F_{\text {R-A }}$.

La surface de charge $F_{\mathrm{R}-\mathrm{A}}$ est une ellipse donnée par l'expression:

$F_{\mathrm{R}-\mathrm{A}}=\left(\mathrm{q}-\alpha_{\mathrm{q}}\right)^{2}+\mathrm{M}^{2}\left(\mathrm{P}^{\prime}-\alpha_{\rho}+\mathrm{a}_{\mathrm{f}}\right)\left(\mathrm{P}^{\prime}-\alpha_{\mathrm{p}}-\mathrm{a}_{\mathrm{p}}\right) \mathrm{R}^{2}(\theta)=0$

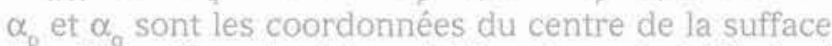
$\mathrm{F}_{\mathrm{R}-\mathrm{A}}^{\mathrm{A}}$. Elles dépendent du point fixe de contact $\left(\mathrm{P}^{\prime}\right.$, et $\left.\mathrm{q}_{1}\right)$ des deux surfaces de charge (surface $F_{\mathrm{A}-\mathrm{A}}$ et ancienne surface) et de l'état de contrainte actuel pendant la décharge ou la recharge ( $\mathrm{P}^{\prime}$ et $\left.\mathrm{q}\right)$.

$$
\begin{aligned}
& \alpha_{\mathrm{p}}=\frac{\mathrm{P}^{\prime}+\mathrm{P}_{1}^{\prime}}{2} ; \alpha_{\mathrm{q}}=\frac{\mathrm{q}+\mathrm{q}_{1}}{2} \\
& \left(\alpha_{\mathrm{p}}=\alpha_{\mathrm{p} 1} ; \alpha_{\mathrm{q}}=\alpha_{\mathrm{q} 1} \text { à l'état initial }\right)
\end{aligned}
$$

a est le paramètre d'écrouissage ; il est le grand rayon de la surface $\mathrm{F}_{\mathrm{R}-\mathrm{A}}$ défini par:

$\mathrm{a}_{\mathrm{i}}=\mathrm{a}_{0}^{\prime}=\mathrm{a}_{0} \exp \left(\frac{2 \mathrm{P}_{0}}{\mathrm{P}_{\mathrm{C} 0}}\right)$ à l'état initial; $\hat{\mathrm{a}}_{1}=\mathrm{B}_{1} \dot{\varepsilon}_{\mathrm{v}}^{\mathrm{p}}+\mathrm{B}_{2} \dot{\varepsilon}_{\mathrm{d}}^{\mathrm{p}}$ avec:

$a_{0}$ : rayon initial de la surface

$\mathrm{P}_{0}$ : la contrainte moyenne initiale

$\varepsilon_{\mathrm{d}}^{\mathrm{p}}$ : la déformation plastique déviatoire

$$
\mathrm{B}_{1}=\mathrm{P}^{\prime} \beta_{2} \frac{\partial \mathrm{F}_{\mathrm{R}-\mathrm{A}} / \partial \mathrm{P}^{\prime}}{-2 \partial \mathrm{F}_{\mathrm{R}-\mathrm{A}} / \partial \mathrm{a}_{\mathrm{i}}} ; \mathrm{B}_{2}=\mathrm{MP}^{\prime} \beta_{2} \frac{\partial \mathrm{F}_{\mathrm{R}-\mathrm{A}} / \partial \mathrm{q}}{-2 \partial \mathrm{F}_{\mathrm{B}-\mathrm{A}} / \partial \mathrm{a}_{\mathrm{i}}}
$$

$\beta_{2}$ : le module de compressibilité plastique à l'état surconsolidé avec:

$\beta_{2}=\beta_{d}=\beta_{1}\left(1+\frac{\left(P_{c}\right)_{\text {ref. }}}{\left(P_{c}\right)_{\text {dech. }}}\right) \frac{P_{c}}{P^{\prime}}$ en décharge

$\beta_{2}=\beta_{r}=\beta_{1}\left(1+\frac{\left(P_{c}\right)_{\text {ref. }}}{P_{c}}\right) \frac{2 P_{c f}}{3\left(P_{c}\right)_{\text {dech. }}} \frac{P_{c}^{\prime}}{P_{c}}$ en recharge

$\left(\mathrm{P}_{\mathrm{C}}\right)_{\text {dech }}$ : la pression de consolidation en décharge, $\left(\mathrm{P}_{\mathrm{C}}\right)_{\text {ef }}$ : paramètre du modèle, représentant la pression de consolidation de référence. Il est à noter que cette pression correspond à celle à partir de laquelle on effectue une décharge dans un essai œdométrique tel que:

$$
\beta_{\mathrm{d}}=\frac{1+\mathrm{e}_{0}}{\lambda_{2}-\kappa}=2 \beta_{1}
$$


Le critère général permettant de différencier le déchargement du rechargement est obtenu à partir du signe de l'incrément du paramètre d'écrouissage ả. Ce dernier doit être nécessairement positif, aussi la présence d'un incrément à négatif au cours du trajet de chargement impose le changement du sens de la sollicitation et l'initialisation d'une nouvelle surface de charge $\mathrm{F}_{\mathrm{B} \cdot \mathrm{A}}$.

Les taux de déformations plastiques volumique et déviatoire sont déterminés par:

$$
\begin{gathered}
\dot{\varepsilon}_{\mathrm{v}}^{\mathrm{p}}=\mathrm{d} \lambda \cdot \frac{\partial \mathrm{F}_{\mathrm{R}-\mathrm{A}}}{\partial \mathrm{P}^{\prime}} ; \dot{\varepsilon}_{\mathrm{d}}^{\mathrm{p}}=\mathrm{d} \lambda \cdot\left(\frac{\partial \mathrm{F}_{\mathrm{R}-\mathrm{A}}}{\partial \mathrm{q}}-\alpha \frac{\partial \mathrm{F}_{\mathrm{H}-\mathrm{A}}}{\partial \mathrm{P}^{\prime}}\right) \\
\alpha=\alpha_{\mathrm{d}}=\left(1,0-\frac{\left|\mathrm{P}_{1}^{\prime}-\alpha_{\mathrm{p} 1}\right|}{\mathrm{a}_{0}^{\prime}}\right)\left(\frac{1,5 \mathrm{P}_{\mathrm{c}}}{\left(\mathrm{P}_{\mathrm{c}}\right)_{\text {dech }}}-0,5\right) \text { en décharge } \\
\alpha=\alpha_{\mathrm{r}}=\left(1,0-\frac{\left|\mathrm{P}_{1}^{\prime}-\alpha_{\mathrm{p} 1}\right|}{\mathrm{a}_{0}^{\prime}}\right)\left(1,0-1,5 \frac{\mathrm{P}_{\mathrm{c}}}{\mathrm{P}_{\mathrm{cf}}}\right) \text { en décharge }
\end{gathered}
$$

d $\lambda$ est déterminé par la condition de consistance; il est donné par la formule suivante:

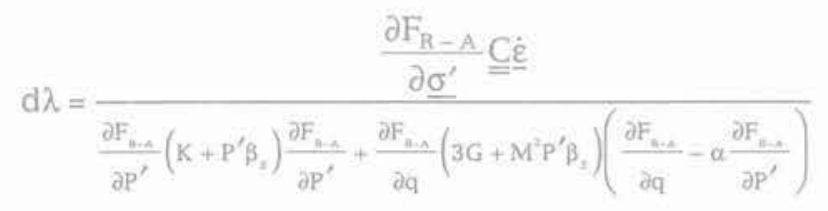

\section{5}

\section{Caractéristique typique des modélisations}

Le modèle proposé nécessite la détermination de huit paramètres rhéologiques, obtenus à partir des essais œedométriques et triaxiaux.

i) Les paramètres correspondant à la surface $F_{C}: K_{a^{\prime}} G_{a^{\prime}}$ $n, P_{\text {co }} \beta_{1}, M$

ii) Les paramètres correspondant à la surface $F_{R-A}: a_{0}$ $\left(\mathrm{P}_{\mathrm{C}}\right)_{\text {ref }}$

Pour l'argile de Boom, les valeurs des paramètres utilisées sont données ci-dessous:

$$
\begin{aligned}
& \mathrm{K}_{\mathrm{a}}=330 \\
& \mathrm{G}_{\mathrm{a}}=150 \\
& \mathrm{n}=1 \\
& \mathrm{P}_{\mathrm{Co}}=6 \mathrm{MPa} \\
& \beta_{1}=18 \\
& \mathrm{M}=0,86 \\
& \mathrm{a}_{0}=0,2 \mathrm{MPa} \\
& \left(\mathrm{P}_{\mathrm{C}}\right)_{\text {tef: }}=10 \mathrm{MPa}
\end{aligned}
$$

Deux chemins ont été étudiés, les figures 5 à 7 présentent la comparaison entre les résultats expérimentaux et les simulations numériques sur cette argile.

\section{Chemin 1: Couplage des deux mécanismes $F_{c}$ et $F_{R-A}$ sur chemin œdométrique cyclique}

La première partie du chargement est réalisée sur le matériau naturel, surconsolidé et entraîne l'activation du mécanisme plastique $\mathrm{F}_{\mathrm{R}-\mathrm{A}}$ (Fig. 5). A partir de l'état de contrainte normalement consolidé $\left(\mathrm{P}_{\mathrm{co}}=6 \mathrm{MPa}\right)$, le premier mécanisme plastique $\mathrm{F}_{\mathrm{C}}$ est substitué à celui de $\mathrm{F}_{\mathrm{B}-\mathrm{A}}$. En décharge, ce dernier mécanisme est réactivé; $\mathrm{i}$ est caractérisé par un module de décharge élevé et une variation très fortement non linéaire du rapport $\mathrm{q} / \mathrm{P}^{\prime}$. Par ailleurs, le chargement cyclique montre une boucle d'hystérésis importante.

\section{Chemin 2: Déformation volumique induite par le méca- nisme $F_{R-A}$ sur des chemins triaxiaux}

Le chemin de contrainte suivi dans l'essai triaxial drainé sur matériau surconsolidé provoque lactivation du deuxième mécanisme plastique $\mathrm{F}_{\mathrm{R} \cdot \mathrm{A}}$. Les déformations volumiques générées par ce mécanisme sont contractantes (Fig. 6). Lorsque l'état de contrainte devient normalement consolidé, les deux surfaces de charges $F_{\mathrm{R}-\mathrm{A}}$ et $\mathrm{F}_{\mathrm{C}}$ sont en contact. La poursuite du chargement s'effectue avec l'activation du premier mécanisme $F_{\text {. }}$. Suivant la position du point de contact des deux surfaces de charge, les déformations volumiques sont contractantes dans le domaine situé en dessous de la droite $\mathrm{q}=\mathrm{MP}$ (Fig. 6b, c). En revanche pour le domaine situé au-dessus de la droite $\mathrm{q}=\mathrm{MP}$, les déformations volumiques sont dilatantes (Fig. 6d). Les boucles d'hystérésis sur chargement cyclique sont d'autant plus marquées que les états de contrainte sont surconsolidés (Fig. 6f). On constate par ailleurs que la pente de décharge augmente avec la contrainte déviatoire de décharge.

\section{Conclusion}

L'inventaire des mécanismes microscopiques physico-chimiques qui gouvernent le comportement macroscopique des argiles gonflantes a été mis à profit pour établir un modèle élasto-plastique actuellement limité aux aspects de la réponse contrainte-déformation. Ce modèle utilise deux variables indépendantes comme contrainte effective. Pour le cas général, il est caractérisé par deux surfaces de charge $F_{C}$ et $F_{\text {R.A }}$. Pour les états de contrainte normalement consolidés, un seul mécanisme plastique est activé, celui correspondant à la surface $F_{c}$ à plasticité associative et à écrouissage positif sous l'effet du chargement mécanique. En revanche pour les états de contrainte surconsolidés, le deuxième mécanisme plastique est défini par la surface $F_{\mathrm{R}-\mathrm{A}}$ à plasticité non associative et à écrouissage physico-chimio-mécanique. Le couplage entre les deux surfaces $F_{C}$ et $F_{A \cdot A}$ est assuré par la déformation volumique plastique totale.

Le modèle est quantifié à partir des chemins œedométriques et triaxiaux pour la détermination de huit paramètres dont six sont relatifs à la surface $F_{c}$. Une comparaison entre les résultats expérimentaux sur l'argile de Boom saturée et les prédictions du modèle montrent que l'ensemble des phénomènes est correctement décrit. En particulier, les déformations volumiques de contractance pour une argile gonflante surconsolidée sur des chemins triaxiaux de recharge et l'existence d'une boucle d'hystérésis très marquée sur des chemins cycliques œdométriques sont parfaitement modélisées. 


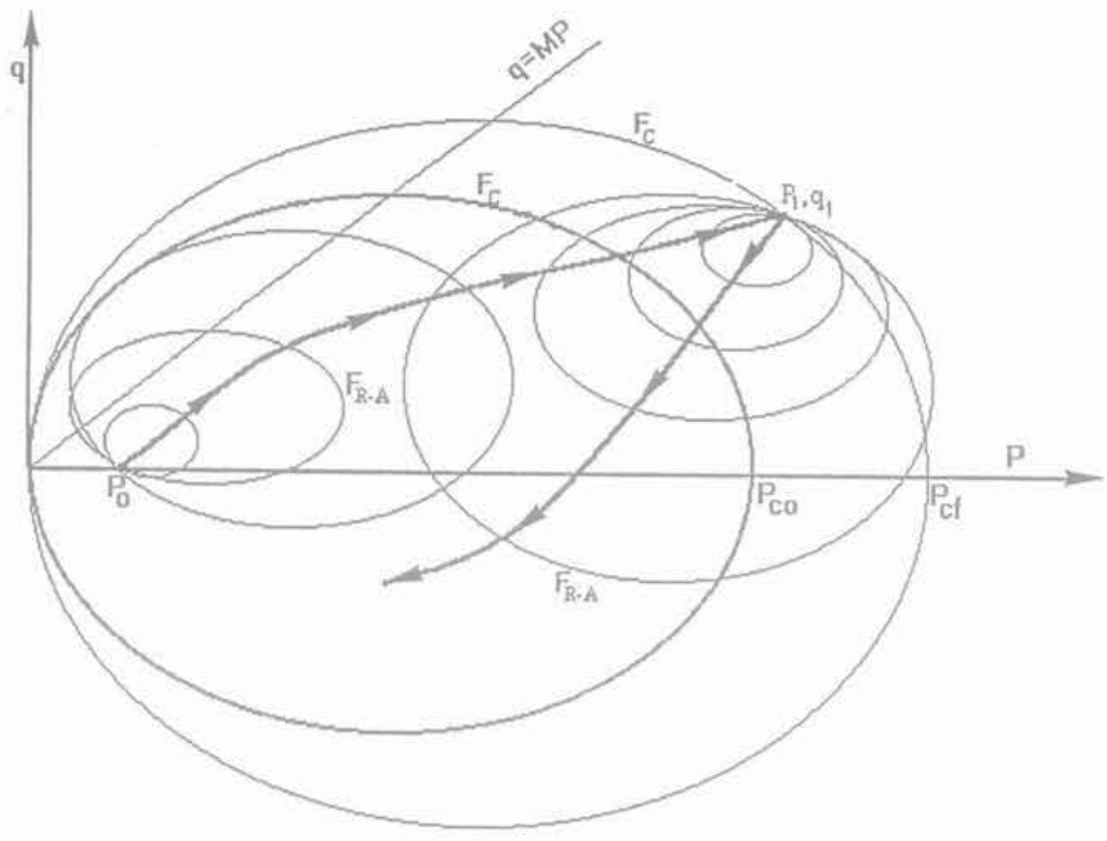

(a)
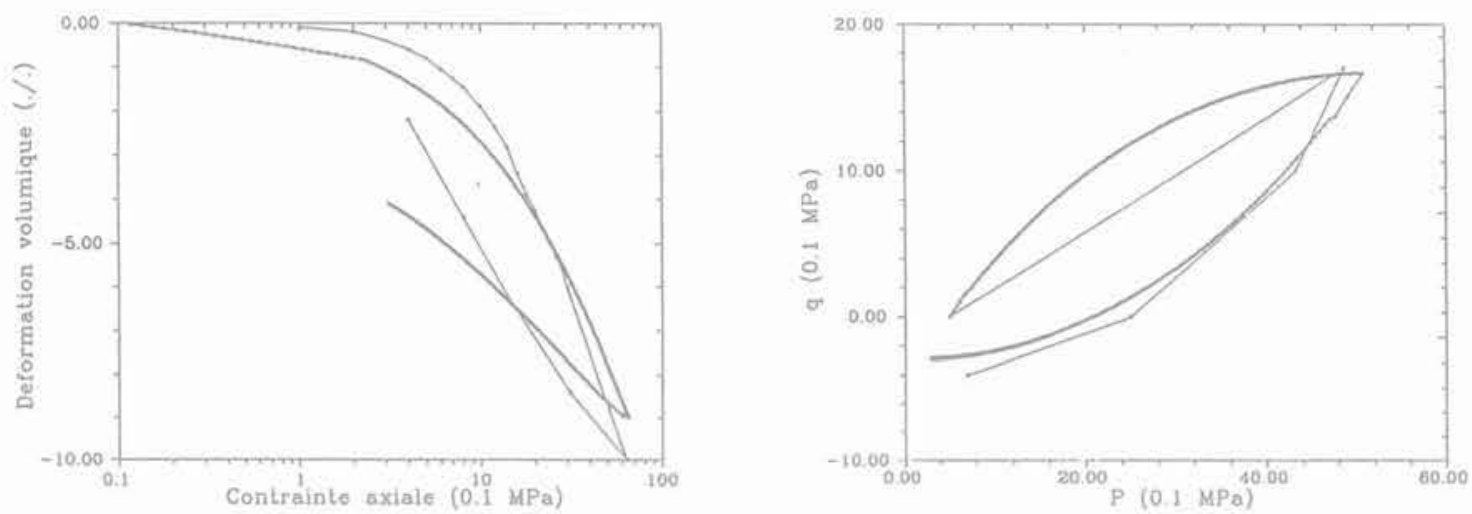

(b)
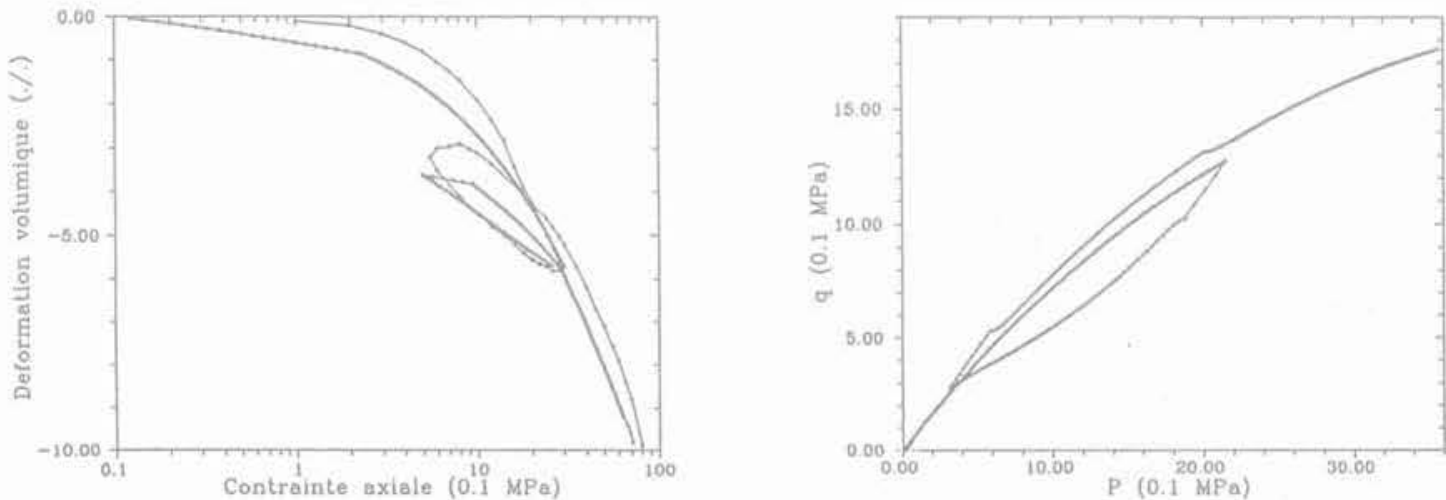

(c)

Expérience (Baldi 1987)

\section{Calcul}

FG.5 Essais cedométriques: (a) Schématisation des surfaces de charge $F_{C}$ et $F_{R-A}$ dans $\mathrm{P}^{\prime}-q$ pendant un cycle "charge-décharge »; (b), (c) Comparaison entre le modèle et l'expérience sur l'argile naturelle de Boom. (Edometer tests: (a) Schematic illustration of vield surfaces $F_{\text {et }} F_{1}$ in plane $P^{\prime}$ - $q$ during "loading-inloading" cycle; (b), (c) Comparison between the model and the tests in natural Boom clay. 


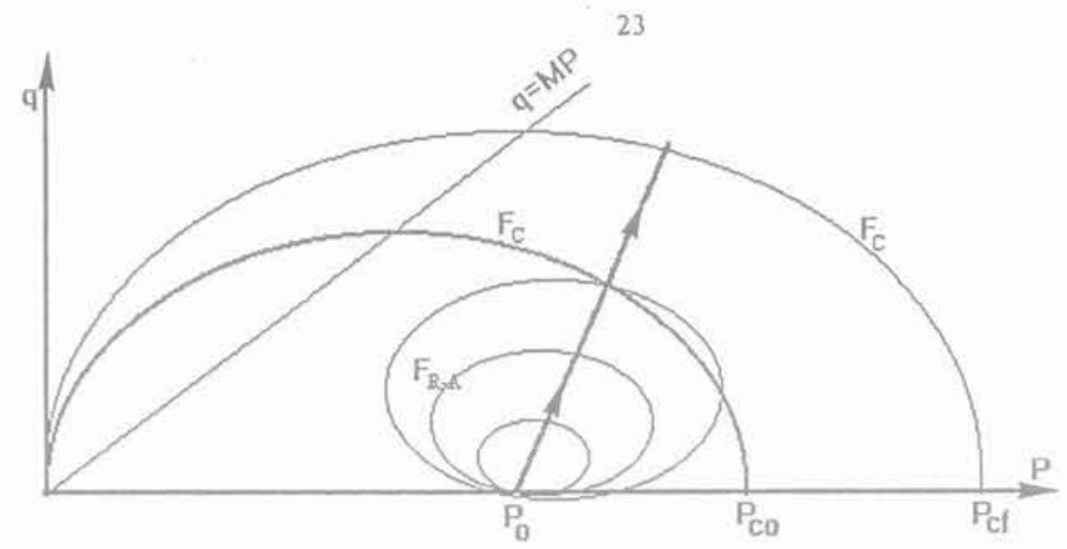

(a)
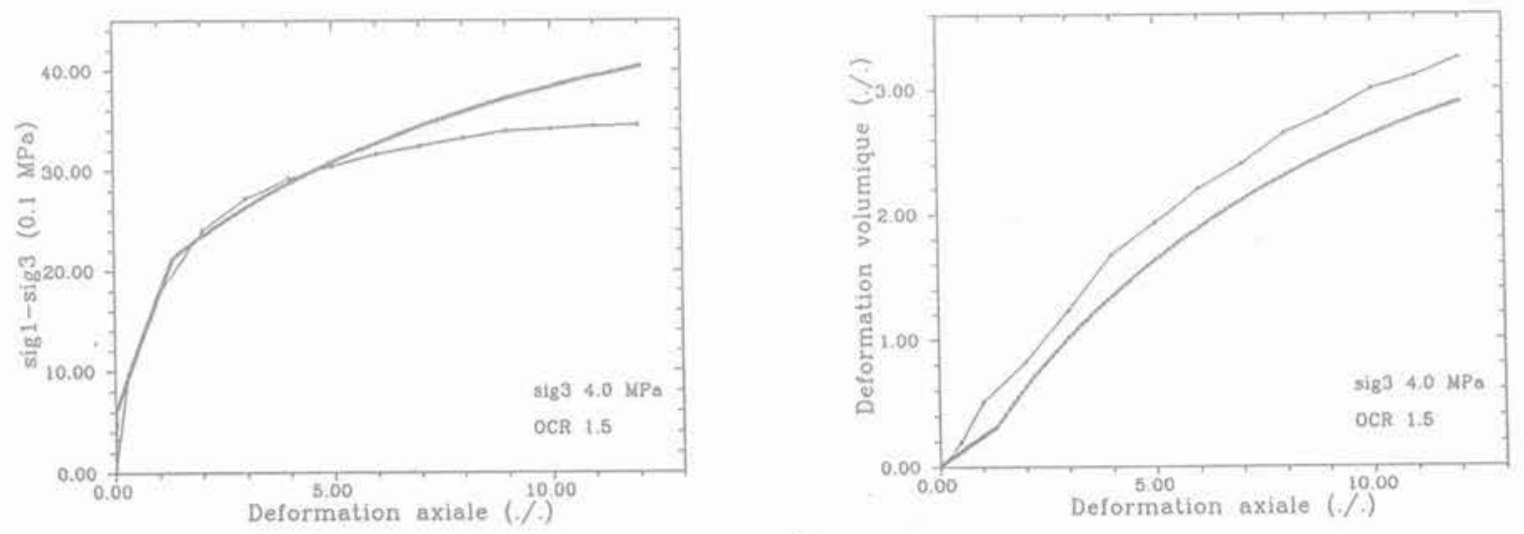

(b)
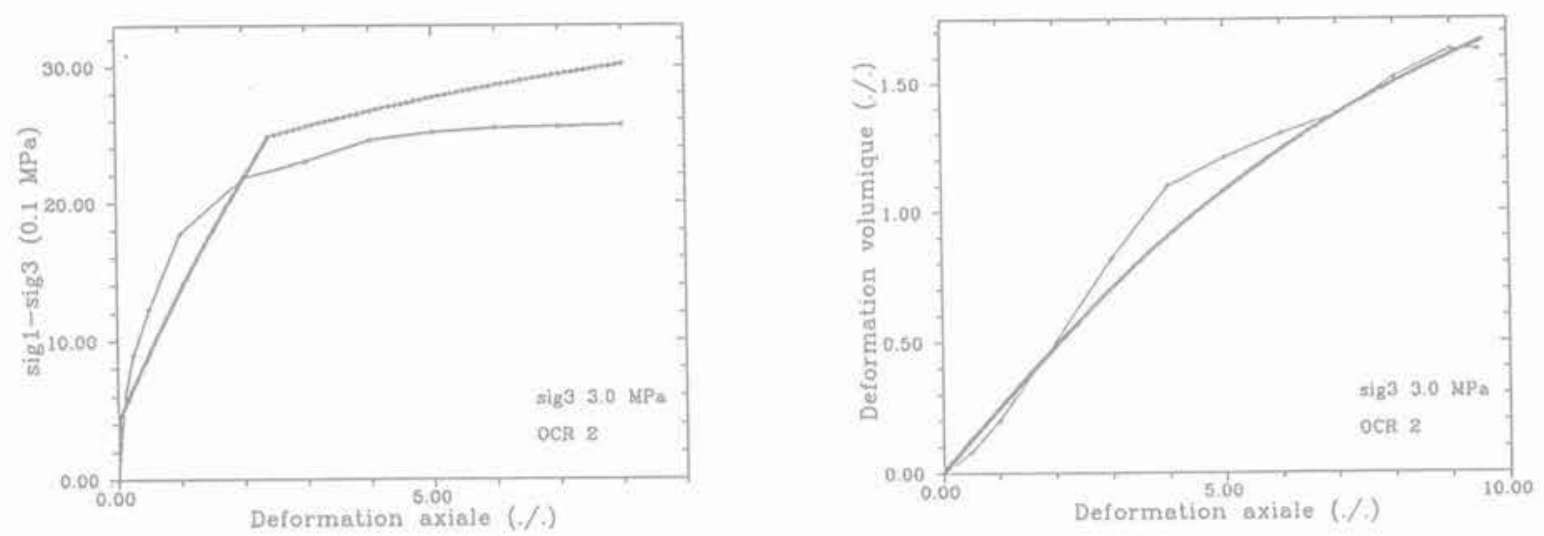

(c)

Expérience (Baldi 1987)

Calcul 

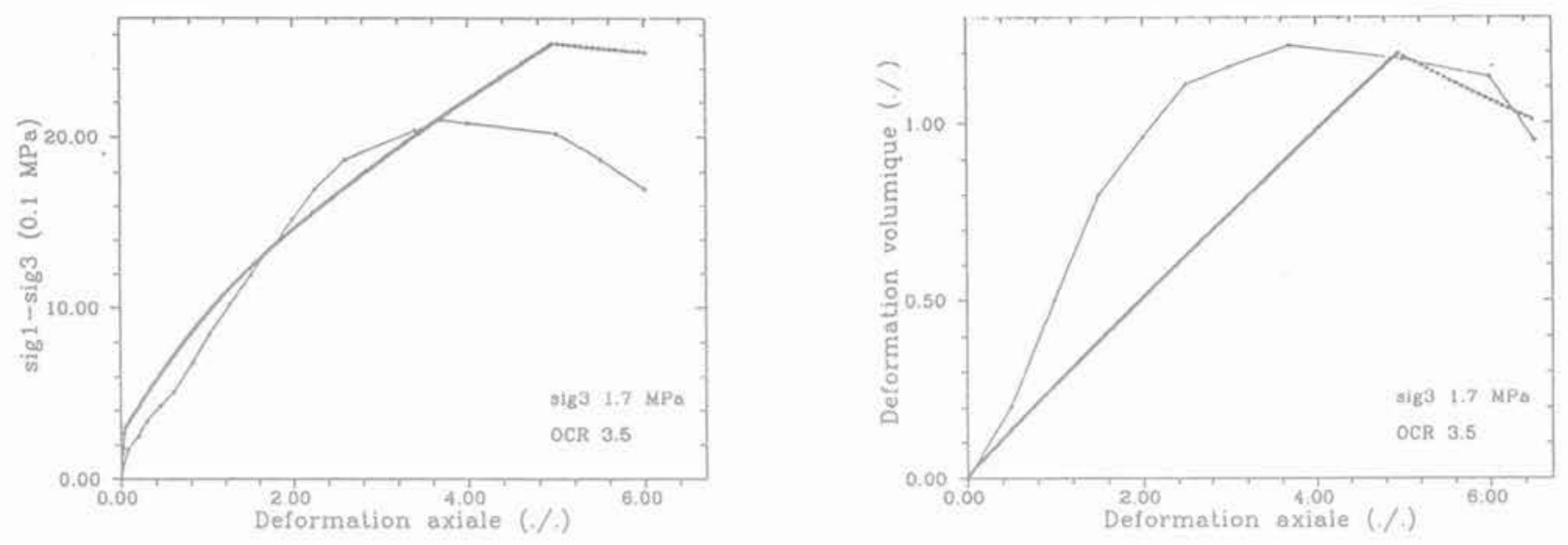

(d)
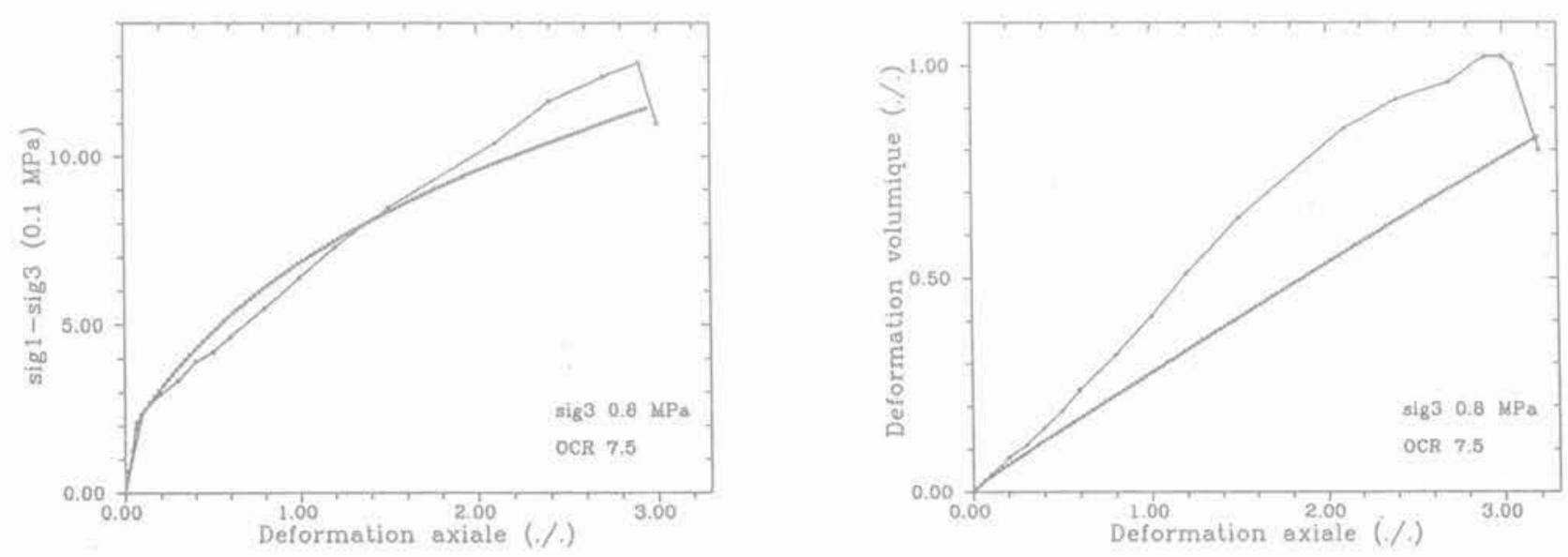

(e)
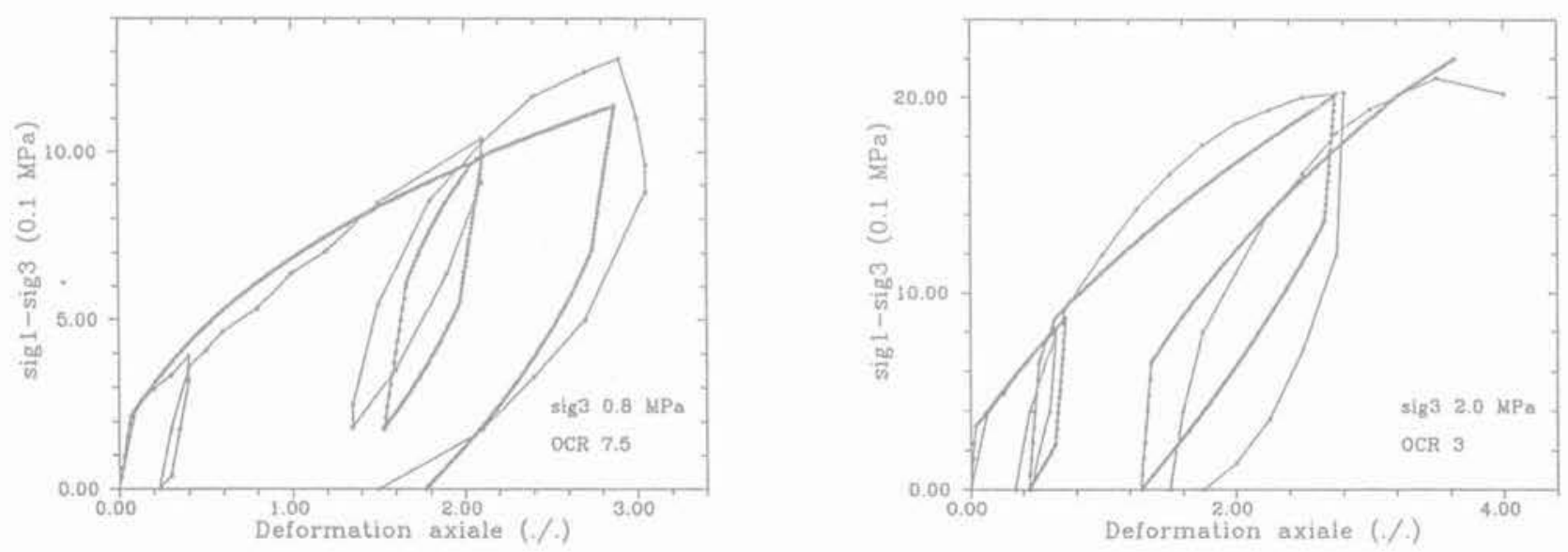

$(\mathrm{f})$

EG.6 Essais triaxiaux drainés: (a) Schématisation des surfaces de charge $F_{C}$ et $F_{k-\Lambda}$ dans le plan $P^{\prime}-q$; (b), (c), (d), (e), (f) Comparaison entre le modèle et l'expérience sur l'argile naturelle de Boom (essais monotones et cycliques).

Drained triaxial tests: (a) Schematic illustration of yield surfaces $F_{C}$ and $F_{R-x}$ in plane $P^{\prime}-q$ :

(b), (c), (d), (e), (f) Comparison between the model and the tests in natural Boom clay (monotonouns and cyclic tests) 


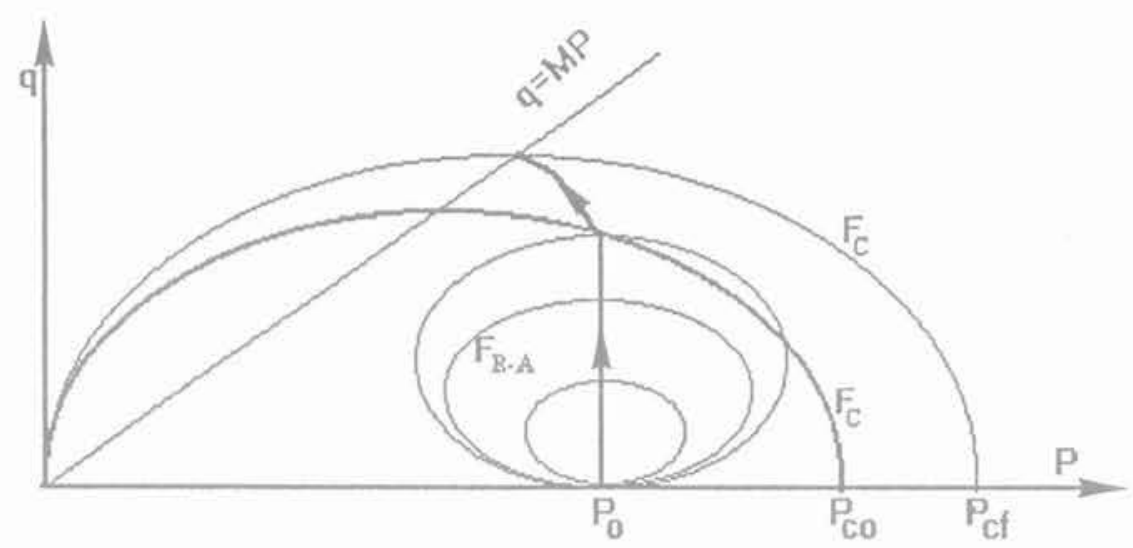

(a)
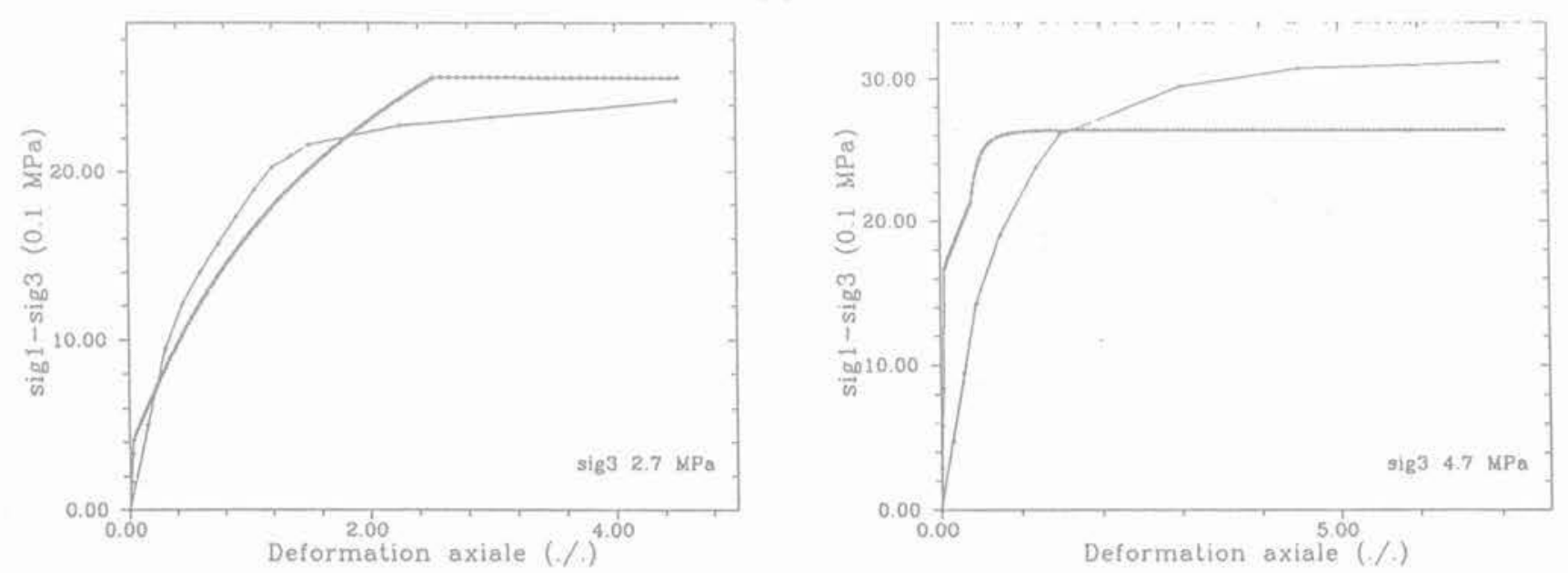

(b)

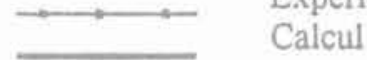

Expérience (Baldi 1987)

Calcul

FiG.7 Essais triaxiaux non-drainés: (a) Schématisation des surfaces de charge $F_{c}$ et $F_{\mathrm{B}-\mathrm{A}}$ dans le plan $\mathrm{P}^{\prime}-\mathrm{q}$; (b) Comparaison entre le modèle et l'expérience sur l'argile naturelle de Boom.

Undrained triaxial tests: (a) Schematic illustration of yield surfaces $F_{c}$ and $F_{p}$ in plane $\mathrm{P}^{\prime}-\mathrm{q}$; (b) Comparison between the model and the tests in natural Boom clay.

\section{Bibliographie}

Atabek R. Felix B., Robinet J.-C, Lahlou R. (1991) - Pheological behaviour of saturated expansive clay materials, Workshop on stress partitioning in engineered clay barriers. Duke University, 29-31 may 1991

Baidi G. Hueckel T., Peano A. Pellegrini R. (1990) - Development in modelling of the thermo-hydro-geomechanical behaviour of Boom Clay and clay based buffer materials, Final report for CEC (FI1W/0150 A), ISMES, Bergamo, Italy.

Bolt G.H. (1956)- Physico-Chemical analysis of the compressibility of pure clays. Géotechnique, 6: 86-93.

Horn R.G., Israelachvili J.N. (1981) - Journal of chemistry physics, 75, 1400-1411.

Hujeux J.-C. (1985) - Une loi de comportement pour le chargement cyclique des sols, in Genie parasismique, Presses de I'ENPC: $287-302$

Israelachvill J.N., Adams G.E. (1978) - Measurement of forces between two mica surfaces in agueous electrolyte solutions in the range $0-100 \mathrm{~nm}$, Journal of the chemical society, Faraday Transactions 1. 74: 975-1001.

Israelachvili J.N. Pashley F.M. (1983)Molecular layering of water at surfaces and origin of repulsive hydration forces. Nature, 306: 249-250.

Low P.F. (1979) - Nature and properties of water in Montmorillonite-Water system. American journal of Soll Science Society, 43: $651-658$.

Martin B.T. (1960) - Adsorbed water on clay, Clay and Clay minerals, Pergamon New York 9:28-70

Mitchell J.K. (1976) - Fundamentals of soil behaviour, John Wiley, New York. Pond G.N., Zienkiewicz O.C. (1982) - Soil mechanics transient and cyclic loads. John Wiley, New York.

Pons C.H., Rousseaux F, Tchoubar D. (1982) - Utilisation du rayonnement synchroton en diffusion aux petits angles pour l'étude du gonflement des smectites, Eau-smectite en fonction de la température, Clay Mineral, 17:327-338. Pusch R. (1982) - Mineral-water interac- tions and their influence on the physical behaviour of highly compacted Na bentonite, Canadian Geotechnical Journal. 19:381-387

Roscoe K.H., Burland J.-B. - On the generalised stress-strain behaviour of "wet" clay. Engineering Plasticity, Editeur: J. Heyman et F.A. Leckie, Cambridge University Press, : 535-609.

Sridharan A. (1990) - Strength and volume change behaviour of a sand-bentonite mixture, Canadian Geotechnical Journal, 27: 404.

Sridharan A.. Jayadava M.S. (1982) Double layer theory and compressibility of clays, Géotechnique, 32: 133-144

Touret O Pons C. H. (1989) - Etude de la répartition de l'eau dans les argiles saturées aux fortes teneurs en eau, Clay Mineral 25: 217-223.

Van Damme H. Bergava F., Gatineau L. (1987) - Contraintes structurales sur la réactivité dans les arqiles et les solides très divisés, Journal de Chimie Physique, $84 n^{\circ} 9,1075-1082$ 